\title{
Los universales y la distinción lockeana entre las esencias nominales y reales ${ }^{1}$
}

Carmen Silva

\section{Introducción}

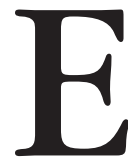

n este artículo abordaremos la perspectiva lockeana respecto de los universales, la cual representa -entre otras cuestiones y desde mi punto de vista- el resultado de la síntesis de dos temáticas muy importantes en su época y que son las siguientes: 1) la naturaleza del lenguaje, y 2) la aplicación del modelo mecánico al conocimiento del mundo natural. En gran medida, la propuesta lockeana gira alrededor de estos dos aspectos cuyo carácter es netamente moderno. Sobre el primero, hablaremos en las secciones iniciales; y, sobre el segundo, en las últimas. Asimismo, creemos que un eslabón entre la reflexión sobre el lenguaje y el mecanicismo es el problema del conocimiento, y en particular -y de suma importancia para los pensadores del siglo XVII-, el del conocimiento de la naturaleza (externa). Además, el conocimiento del mundo natural concierne a la empresa fundamental del proyecto moderno: elaborar una nueva filosofía natural como alternativa al modelo explicativo aristotélico ya en decadencia. Como vemos, dentro de este planteamiento se inscriben tanto la reflexión sobre el lenguaje como el mecanicismo; ambos, en el caso de Locke, representan las dos etapas o momentos de su posición sobre los universales, asunto que analizaremos en las siguientes páginas.

\section{La importancia del lenguaje en el pensamiento moderno}

Para hablar sobre el lenguaje en el pensamiento moderno, tomamos como referencia el artículo de Marcelo Dascal titulado: "Lenguaje y conocimiento

${ }^{1}$ Una versión previa de este trabajo fue leída en el XV Congreso Internacional de Filosofía: "El diálogo filosófico", organizado por la Asociación Filosófica de México y la 
en la filosofía moderna", en el que el autor señala un conjunto de cuestiones sobresalientes para comprender la relación que los filósofos del XVII establecieron con el lenguaje. El primero que menciona es el siguiente:

La modernidad se instaura, como es sabido, por un rompimiento declarado con lo anterior, lo "viejo", especialmente con la escolástica medieval y con el neoplatonismo y el aristotelismo renacentistas. Como veremos, esa ruptura es profunda y se manifiesta en una nueva confirmación del saber y de los modos de pensar, en nuestro caso, de pensar el lenguaje y su rol en el conocimiento. Sin embargo, las rupturas no son jamás totales, y aun si se desechan los antiguos marcos, los viejos temas no desaparecen de las telas modernas.

El siglo XVII tratará con enorme intensidad la mayoría de los temas "lingüísticos" que han preocupado a los siglos anteriores, pero de forma distinta. Incontestablemente se puede decir que ese siglo se siente inmensamente atraído por el lenguaje. A la vez, sin embargo, le teme y lo critica violentamente. ¿Por qué tanto interés por lo lingüístico? ¿Por qué esa actitud en que mezclan atracción y repulsión, fascinación y temor? ${ }^{2}$

Según lo que Dascal ha afirmado anteriormente, la relevancia de los temas lingüísticos en el siglo XVII se debe a que en esa época el objeto de interés primordial era el conocimiento. Los propios representantes de este momento histórico reconocen en forma clara la estrecha relación entre conocimiento y lenguaje, y por ello la lengua es objeto de sus reflexiones centrales. De nuevo cito un pasaje de Dascal muy sugerente:

Lo que caracteriza las actitudes de los pensadores de la edad moderna frente al lenguaje es, pues, más que cualquier otro factor, su preocupación por la relación lenguaje-conocimiento: [y por ello se preguntan] ¿ayuda el lenguaje a promover el saber o impide su progreso?, ésta es la cuestión fundamental que se plantea. Es esta la cuestión que determina el orden del día de la filosofía del lenguaje en la época, la cuestión a la que se subordinan en realidad todas las demás. En el siglo XVII, Bacon, Hobbes, Descartes, Locke, Spinoza, Leibniz, Arnauld se interesan todos

Universidad Nacional Autónoma de México, en el simposio "Filosofía moderna: ontología y escepticismo", en la ciudad de México el 29 de enero de 2010. Agradezco a los árbitros sus atinados comentarios.

${ }^{2}$ Marcelo Dascal, "Lenguaje y conocimiento en la filosofía moderna", en Ezequiel de Olaso, ed., Del Renacimiento a la Ilustración I. Madrid, Trotta/Consejo Superior de Investigaciones Científicas, 1994, p. 15. (Enciclopedia iberoamericana de filosofía, 6) 
por el rol - positivo o negativo- del lenguaje en el conocimiento. Sus contribuciones al tema elaboraron una gama de posiciones y argumentos que el siglo XVIII retoma y profundiza, siempre en el marco de la cuestión central del rol cognoscitivo del lenguaje, como lo prueban las reflexiones sobre el lenguaje de Berkeley, Hume, A. Smith, Rousseau, Condillac, Herder, los Enciclopedistas [... $]^{3}$

En esta parte el autor define muy bien la importancia del lenguaje para los filósofos del XVII y cómo esta consideración la retoman los ilustrados.

A continuación citaremos dos cuestiones: la primera es el contexto histórico propio del XVII, el cual nos ayuda a comprender por qué la preocupación sobre el lenguaje fue tan destacada, además de la del conocimiento que ya mencionamos. La segunda es una lista de temas sobre el lenguaje o relacionados con él y que los autores del periodo tenían muy presente. Veamos la primera, expuesta por Dascal en el siguiente párrafo:

Las principales causas que llevaron al siglo XVII a ocuparse tan obsesivamente del conocimiento se encuentran en los procesos históricos de la época que problematizaron antiguas costumbres; la reforma y la contrarreforma, la crítica a la ciencia aristotélica, a la escolástica y a las corrientes de pensamiento renacentistas, la crisis del antiguo sistema educativo, la difusión del escepticismo, y las necesidades creadas por la expansión geográfica, tecnológica y económica de Europa, así como por su diversificación interna en unidades nacionales. Si se agregan a esta lista los cambios propiamente lingüísticos (por ejemplo, el ascenso de los idiomas vernáculos y el correspondiente descenso del latín, la confrontación con las lenguas recién descubiertas de las Américas, etcétera), tenemos un panorama de los factores que exigen cambios radicales también en la filosofía del lenguaje. ${ }^{4}$

A pesar de los cambios que ocurrieron en la sociedad europea del XVII y que explican la razón de que el lenguaje sea una disertación esencial para los filósofos modernos, Dascal insiste en señalar que: "la temática lingüística 'anterior' no desaparece del pensamiento de la edad moderna". ${ }^{5}$ Los temas que transitan entre una y otra época son los siguientes: 1) la cuestión del origen de las leguas, 2) la pregunta sobre su naturalidad o convencionalidad, 3) el problema de los universales, 4) el debate sobre la interpretación, 5) las relaciones

\footnotetext{
${ }^{3}$ Ibid., pp. 15-16

${ }^{4}$ Ibid., pp. 16-17.

${ }^{5}$ Ibid., p. 17.
} 
entre lógica y gramática, 6) y entre dialéctica y retórica, 7) el interés por las escrituras secretas y la criptografía en general, 8) el rechazo y aceptación de la creencia en el poder secreto de las palabras, por ejemplo: la cábala, la magia y la alquimia, 9) la cuestión de la normatividad del lenguaje, 10) el anhelo por recobrar la unidad perdida del lenguaje ${ }^{6} \mathrm{y}$, por último, 11) la investigación sobre la naturaleza y valor del lenguaje matemático y su aplicación a la nueva filosofía natural. ${ }^{7}$

Por tanto, en esta parte podemos concluir que Dascal nos ofrece en este artículo un panorama muy claro y bien definido de las razones que hacen que el análisis del lenguaje sea crucial para los filósofos del siglo XVII; y las características que enlista nos permiten detectar la diferencia entre esta filosofía del lenguaje y la medieval. Todo esto nos sirve de marco teórico contextual de la filosofía del lenguaje de John Locke, representante de este periodo del pensamiento filosófico.

\section{La teoría del lenguaje de John Locke}

\section{Funciones del lenguaje}

Para Locke, en su Ensayo sobre el entendimiento humano, el lenguaje tiene dos funciones fundamentales: en primer lugar, la de comunicar las ideas o contenidos mentales, necesidad relacionada con su propia teoría de las ideas, la cual fácilmente puede llevarnos al solipsismo. En segundo lugar, la de apoyar al conocimiento, referida anteriormente por Dascal. Nosotros creemos que Locke sostenía tanto la primera como la segunda concepción; es decir, creía que el lenguaje era un medio de comunicación y un apoyo al conocimiento.

Propongo que ahora prosigamos acercándonos a la filosofía del lenguaje del autor inglés, y de esa forma podamos apreciar sus características, objetivos, logros y dificultades. ${ }^{8}$

${ }^{6}$ En su listado, Dascal no la menciona, pero un elemento importante para la reflexión sobre el lenguaje es la distinción entre el hombre y los otros seres vivos.

${ }^{7}$ Para leer lo que comenta el autor sobre cada uno de los incisos antes numerados, véase M. Dascal, op. cit., p. 17.

${ }^{8}$ Sobre las dificultades de la teoría del lenguaje de Locke no hablaré en esta primera versión del trabajo, pues lo que aquí me interesa exponer con mayor claridad es su peculiar e innovadora concepción frente al tema de los universales. 


\section{Lenguaje y comunicación: necesidad y posibilidad exclusivamente humanas}

En esta sección veremos las características de la teoría del lenguaje de John Locke sin perder de vista nuestra prioridad, que es el tema de los universales (y quizá su punto de partida), presentado por vez primera cuando el autor, en su reflexión sobre el lenguaje, habla de las palabras generales. Antes de abordar estos conceptos cabe referirnos a lo que señala el filósofo cuando dice que el lenguaje es algo propio y exclusivo de los seres humanos. Sobre ello versa el siguiente pasaje:

Dios decidió que el hombre fuera una criatura sociable, lo hizo no sólo con la inclinación y la necesidad de relacionarse con los de su propia especie, sino que además lo dotó de un lenguaje, que sería un gran instrumento y vínculo común con la sociedad. Por ello, el hombre tiene por naturaleza los órganos adecuados de tal manera que está en disposición de emitir sonidos articulados a los que llamamos palabras. Pero esto todavía no es suficiente para producir el lenguaje, pues los loros, y otros pájaros, pueden ser adiestrados para que produzcan sonidos articulados diferentes, y, sin embargo, esto no quiere decir que estén en posesión del lenguaje.?

Mediante estas ideas advertimos que los sonidos articulados no son exclusivos del hombre, pues otros seres vivos, en particular ciertas aves, los pueden emitir. La cuestión es, ¿qué hace que los sonidos articulados de los hombres sean diferentes a los de cualquier otro animal? A hora leamos lo que el filósofo

${ }^{9}$ John Locke, An Essay Concerning Human Understanding, 2 vols. Ed. de Alexander Fraseer. Nueva York, Dover, 1959, III, i, 1. (En las referencias al Ensayo, según la citación clásica, los diferentes números indican lo siguiente: libro, capítulo y sección. A continuación nos referiremos a esta obra traducida al español como el Ensayo sobre el entendimiento humano). En diferentes partes del Ensayo, el autor menciona a los pájaros parlantes que emiten sonidos articulados. Esto denota las repercusiones que tuvo en Europa el descubrimiento de nuevas tierras en las que existían seres humanos con rasgos físicos, creencias religiosas, animales y alimentos desconocidos anteriormente. Un ejemplo de esto es el del pájaro parlante, el cual supongo es propio de tierras con climas más cálidos que las europeas. Otro aspecto utilizado por Locke es el del sabor de la piña, que sólo se puede conocer probándola directamente (fruta que antes y ahora es típicamente tropical). Dicha alusión le sirve para apoyar su idea de que el origen de las ideas sensibles se encuentra en la experiencia. De igual manera, la referencia a otras culturas humanas con otras creencias religiosas es una prueba contra el innatismo. Con todo esto quiero mostrar que el Ensayo es, entre otras cosas, un buen ejemplo de la influencia que tuvo la expansión geográfica en las conciencias europeas. 
inglés responde a esta interrogante: "[...] además de estos sonidos articulados se hizo necesario que el hombre fuera capaz de usarlos como signos de sus concepciones internas; y que estos sonidos se pudieran establecer como señales de las ideas alojadas en su mente, de tal manera que los pensamientos de las mentes de los hombres se comunicaran unas a otras". ${ }^{10}$

La relación que Locke establece aquí entre los sonidos y las ideas muestra claramente cómo el lenguaje es la vía que John Locke propone para superar el solipsismo. Gracias a que la función del lenguaje es comunicar las concepciones internas (propias de cada mente individual), es que el filósofo inglés puede trazar una distinción entre el lenguaje como exclusivamente humano y los sonidos articulados de ciertas aves. Esto mismo se relaciona con el objetivo del lenguaje, que en palabras del autor es el siguiente:

Cuando un hombre se dirige a otro, es para que éste le entienda; y la finalidad del que habla consiste en que aquellos sonidos puedan, en cuanto señales, dar a conocer sus ideas al oyente. Resulta por tanto, que las palabras son las señales o signos de las ideas del hablante y nadie puede aplicarlas, como señales, a ninguna cosa inmediatamente, sino es a las ideas que él mismo tiene. ${ }^{11}$

En este párrafo, y en los que le siguen del libro III, "De las palabras", Locke expone sus ideas acerca del vínculo que hay entre las ideas y el lenguaje, de tal manera que, desde su punto de vista, es imposible que exista una palabra sin antes haber una idea a la cual represente y, por ello, de la que es signo. Así es como establece su teoría del lenguaje en la que las palabras comparten con las ideas una misma "esencia" o estructura, pues ambas son de naturaleza empirista. Acerca de esto Locke escribe lo siguiente: "Un hombre no puede hacer de sus palabras los signos o cualidades de las cosas, o de las concepciones en la mente de los otros hombres, si él mismo no tiene ninguna idea de ello". ${ }^{12}$

Antes de terminar esta sección consideramos necesario referirnos a la definición que propone el pensador inglés acerca de las palabras, las denomina "los signos sensibles" de las ideas en la mente del hablante y creemos que las llama así por la siguiente razón, ya que para él: "el uso de las palabras consiste en que sean las señales sensibles de las ideas, y las ideas que se significan por aquéllas son su significación propia e inmediata". ${ }^{13}$

${ }^{10}$ Ibid., III, i, 2. (Las cursivas son mías).

${ }^{11}$ Ibid., III, ii, 1. (Las cursivas son mías).

${ }^{12}$ Ibid., III, ii, 2.

${ }^{13}$ Ibid., III, ii, 1. 


\section{Comunicación y palabras generales}

La función comunicativa del lenguaje consistente en transmitir las ideas que tengo en mi mente a otro sujeto o mente, es una de las más importantes y a su vez el rasgo que permite distinguir el lenguaje humano de los meros sonidos articulados que pueden producir otros seres vivos. Ahora bien, vayámonos acercando al tema de los universales con otras indicaciones que añade Locke respecto de la comunicación cuando afirma que para que ésta sea posible es necesario que las palabras sean generales. Leamos lo siguiente:

No es suficiente para la perfección del lenguaje con que los sonidos se puedan convertir en signos de ideas, a no ser que esos signos se puedan usar de manera tal que puedan abarcar varias cosas particulares: la multiplicación de las palabras habrían confundido su uso, si cada cosa concreta necesitara de un nombre distinto que la significara. Para remediar este inconveniente, el lenguaje ha tenido un mayor perfeccionamiento en el uso de términos generales, por el que una palabra se hizo para señalar una gran cantidad de existencias particulares; este uso ventajoso de los sonidos se obtuvo solamente por la diferencia de las ideas de las que ellos eran signos, convirtiéndose así esos nombres en generales, los cuales se han hecho para establecer las ideas generales $[\ldots]^{14}$

Es así como Locke plantea que para que la comunicación sea posible la mayoría de las palabras que conforman el lenguaje necesitan ser generales, a pesar de que las cosas existentes que ellas representan sean todas ellas, o en su mayoría, de naturaleza particular. El filósofo habla sobre este tema en los siguientes términos:

Siendo particulares todas las cosas existentes, tal vez sea razonable el considerar que las palabras, que deben conformarse a las cosas, también lo sean -me refiero a su significado-; sin embargo, vemos que sucede lo contrario. La mayor parte de las palabras que forman todos los lenguajes son términos generales; lo cual no ha sido efecto de la negligencia o de la fortuna, sino de la razón y la necesidad. ${ }^{15}$

En efecto, lo que posibilita que el lenguaje sea un medio de transmisión de las ideas que tengo en mi mente a otra mente (o sujeto pensante) son las

\footnotetext{
${ }^{14}$ Ibid., III, i, 3. (Las cursivas son mías).
}

${ }^{15}$ Ibid., III, iii, 1. 
palabras, cuestión que ya señalamos anteriormente. Ahora bien, esto sólo es posible si realmente las palabras son generales y no particulares, pues de otra forma la comunicación resultaría muy difícil y muy limitada. Las razones que ofrece Locke, en defensa de las palabras generales como las que posibilitan la comunicación, son tres y las expone de la siguiente manera:

[En primer lugar] está por encima del poder humano la capacidad de forjar y retener ideas distintas de todas las cosas particulares con las que entramos en contacto $[\ldots]^{16}$

En segundo lugar, si fuera posible, sería inútil, ya que no serviría al fin principal del lenguaje. En vano los hombres amontonarían nombres de cosas particulares, que no les servirían para nada al comunicar sus pensamientos. ${ }^{17}$

En tercer lugar, y aun admitiendo que esto resulta factible (lo que no creo), convendría además advertir que un nombre para una cosa particular no sería de gran utilidad para el conocimiento, el cual, aunque esté fundado en las cosas particulares, se amplía por concepciones generales, a las que las cosas quedan sujetas, una vez reducidas a clases bajo nombres genéricos. ${ }^{18}$

\section{Palabras generales y esencias nominales: una convención}

Una vez que el filósofo ha establecido que las palabras generales son necesarias para la comunicación y el conocimiento, lo primero que debe de explicar es cómo se elaboran estas palabras, para posteriormente dar una respuesta sobre su naturaleza. Veamos lo que afirma sobre ambas cuestiones. En estas citas se ocupa de la elaboración de las ideas generales: "Las palabras llegan a ser generales por ser signos de ideas generales; y las ideas se convierten en generales cuando se separan de las circunstancias de tiempo y lugar y de cualquier otra idea que pueda determinarlas a ellas a esta o aquella existencia particular". ${ }^{19}$

Y la siguiente,

De las ideas complejas significadas por los nombres: hombre y caballo, dejando al margen solamente aquellas particularidades en las que di-

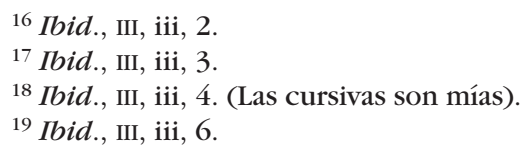


fieren, y manteniendo aquellas otras en las que coinciden, con las que se forja una nueva y distinta idea compleja a la que se da el nombre de animal, logramos un término más general que abarca a los hombres y a otras criaturas. Dejemos a un lado las ideas de animal, sentidos y movimiento espontáneo, y la idea compleja resultante, formada por las ideas simples remanentes de cuerpo, vida y alimento, deviene en una idea simple todavía más general que se engloba bajo el término más comprensivo de ser vivo. Y para no alargarnos más en este particular, tan evidente por sí mismo, es por idéntico camino por el que la mente procede hacia las ideas de cuerpo, de sustancia y, por fin, a las de ser, cosa y otros tales términos universales que significan todas nuestras ideas, cualesquiera que éstas sean. Para terminar con todo este misterio de los géneros y las especies, que tanto ruido meten en las escuelas, y que, con justicia, tan poca atención reciben fuera de ellas, ya que no son nada más que ciertas ideas abstractas, más o menos comprensivas, con nombres anexos a ellas. ${ }^{20}$

Los dos pasajes anteriores son muy importantes porque muestran cómo se forman las palabras generales; las cuales son, en gran medida, el resultado del ejercicio de una facultad del entendimiento llamada abstracción. Pero sigamos ahora en este mismo camino, cuando el autor afirma claramente que las palabras generales, signos sensibles de las ideas abstractas, no son reales, pues son creaciones del entendimiento humano, es decir, convenciones.

Al regresar a las palabras generales, es claro, por todo lo que se ha dicho, que lo general y lo universal no pertenecen a la existencia real de las cosas, sino que son invenciones y criaturas del entendimiento, fabricadas por él para su propio uso, y referidas tan sólo a los signos, sean palabras o ideas. Como ya se dijo, las palabras son generales cuando se usan como signos de ideas generales, y de esta manera se aplican indirectamente a muchas cosas particulares [...] la universalidad no pertenece a las cosas mismas, todas las cuales son particulares en su existencia $[\ldots]^{21}$

\section{Las clases corresponden a nombres}

La posición de Locke sobre el debate alrededor de la naturaleza de los universales, incluidos los géneros y las especies, ya quedó establecida en la sección

${ }^{20}$ Ibid., III, iii, 9.

${ }^{21}$ Ibid., III, iii, 11. (Las cursivas son mías). 
anterior cuando afirma que: "lo general y lo universal no pertenecen a la existencia de lo real", pues, en palabras del autor, "son invenciones y criaturas del entendimiento". ${ }^{22}$

Ésta es la tesis que propone el filósofo inglés frente a las dos corrientes dominantes e irreconciliables en la Edad Media, a saber, la realista y la nominalista. Sin embargo, el autor del Ensayo, en la primera etapa de su reflexión sobre los universales, se define como nominalista, postura propia de los primeros capítulos del libro III. Para mayor claridad sobre el tema, sugiero que leamos el siguiente fragmento:

Para terminar, he aquí algunas cosas que quisiera decir muy brevemente. Todo este gran asunto de los géneros y las especies, y sus esencias, se reduce sólo a esto: que los hombres que forman ideas abstractas, y las fijan en sus mentes con sus nombres, se capacitan de ese modo para considerar las cosas y discurrir sobre ellas [...] para comunicar de manera más fácil y rápida sus conocimientos, los cuales avanzarían muy lentamente si sus palabras y pensamientos estuvieran limitados sólo a lo particular. ${ }^{23}$

Los pasajes de esta sección y la anterior nos muestran que para el autor del Ensayo los universales no corresponden o representan ningún tipo de clase natural, en el sentido de que éstos se refieran a esencias de las cosas o a esencias compartidas (o a clases o grupos de particulares). Esta perspectiva nos permite apreciar una gran coherencia y consistencia en la doctrina empirista de Locke expuesta en la obra citada. En ella, seguidora de la "vía media" propuesta por Mersenne y Gassendi en cuanto al escepticismo pirrónico, rechaza el conocimiento de las esencia y, por tanto, desemboca de manera natural en una postura nominalista sobre la naturaleza de los universales. ${ }^{24}$

\section{Los universales no representan clases naturales}

La primera consecuencia de la afirmación lockeana de que las ideas generales, las abstractas y los universales son construcciones de los hombres -resultado del ejercicio de la facultad de abstraer-, es que las clases que los universales representan son sólo nombres y, por tanto, no son naturales o reales, sino artificiales. Para ejemplificar esta cuestión, transcribo a continuación dos párrafos

${ }^{22}$ Idem .

${ }^{23}$ Ibid., III, iii, 20. (Las cursivas son mías).

${ }^{24}$ Para aquellos interesados en conocer la "vía media" de Mersenne y Gassendi, léase el capítulo VII titulado "El escepticismo constructivo o mitigado", en Richard Popkin, Historia del escepticismo desde Erasmo a Spinoza. México, FCE, 1983. 
del autor: "[...] lo que las palabras generales significan es una clase de cosas; y cada una de ellas significa eso por ser el signo de una idea abstracta que tenemos en la mente, y dicha idea, en tanto las cosas existentes se conforman a ella, caen bajo ese nombre o, lo que es igual, son de esa clase". ${ }^{25}$

La segunda es que estas esencias nominales o ideas generales abstractas, que representan clases o grupos de seres o de cosas, no corresponden a ninguna esencia o estructura de la naturaleza, ya que son el resultado de la percepción, por parte de los hombres, acerca de las semejanzas entre los seres (naturales o artificiales) que los sujetos humanos perciben. En palabras del autor leemos lo siguiente: "No [...] niego, que la naturaleza, al producir las cosas, generó que muchas de ellas resultaran semejantes [...] [y, gracias a ello] podemos afirmar que su clasificación bajo ciertos nombres es un producto del entendimiento, motivado por la similitud que observa entre ellos $[\ldots]^{26}$

\section{Distinción entre esencias nominales y reales}

Después de todas estas afirmaciones sobre el origen y naturaleza de las palabras generales (universales), parecía que el filósofo inglés se reconocía claramente como representante y partidario del nominalismo. Sin embargo, ahora nos da una gran sorpresa con la distinción entre esencias nominales y reales. Pero, adelantándome a la cuestión, diré que, de acuerdo con el autor, las esencias nominales son todas las que conocemos ahora (me refiero a su época) y gracias a las cuales nos comunicamos y podemos clasificar lo existente en clases. Para el filósofo inglés la esencia nominal es:

[...] habiéndose ocupado largamente en las enseñanzas y disputas de las escuelas de los géneros y las especies, la palabra esencia ha llegado casi a perder su significado original, y de esta manera, en lugar de aplicarla a la constitución real de las cosas, ha sido aplicada casi exclusivamente a la constitución artificial de los géneros y las especies. ${ }^{27}$

Vale la pena comentar que para Locke, ferviente seguidor del mecanicismo de su época, por constitución real de una cosa entiende su estructura atómica, algo muy cercano a lo que hoy conocemos como Tabla periódica de los elementos. Pero continuemos con las citas lockeanas sobre las esencias en sus diferentes significados:

${ }^{25}$ J. Locke, op. cit., III, iii, 12.

${ }^{26}$ Ibid., III, iii, 13. (Las cursivas son mías).

${ }^{27}$ Ibid., III, iii, 15. (Las cursivas son mías). 
[...] siendo evidente que las cosas se clasifican bajo nombres en clases o especies, tan sólo cuando se conforman con ciertas ideas abstractas, a las que hemos anexado esos nombres, la esencia de cada género o clase llega a no ser otra cosa que la idea abstracta significada por el nombre general o clasificador [...] Estas dos clases de esencias supongo que no estarán mal designadas si a la una la denomino real y a la otra nominal. ${ }^{28}$

Ahora nos parece pertinente abordar en el siguiente apartado lo que entiende el autor del Ensayo por las esencias reales y si éstas son cognoscibles.

\section{Las esencias reales y el conocimiento}

Es importante anotar que Locke creía en la existencia de las esencias reales, pero, al definirlas como estructuras atómicas, se ve obligado a afirmar que por el momento son desconocidas, mas que es posible que con el avance del conocimiento podamos acceder a ellas. ¿Entonces, las nominales tienen vigencia e importancia mientras avanza el conocimiento? ¿Locke es un nominalista transitorio, en espera de que el desarrollo del conocimiento devele los verdaderos secretos y estructuras del mundo?

Antes de responder a las preguntas planteadas, considero necesario señalar que su realismo no es metafísico, es químico o físico, y esto es relevante porque nos permite mantener la idea antes mencionada de que el Ensayo es una obra consistente y fiel a la "vía media" de Mersenne y Gassendi (y al mecanicismo del segundo) y de Robert Boyle, que es compatible con todo esto.

Para concluir mi presentación, y en particular el asunto de las esencias reales cito a continuación un largo pasaje de Locke, del libro IV, "Del conocimiento":

Si una gran parte o, mejor dicho, la casi totalidad de las diversas especies de cuerpos del universo escapan a nuestros conocimientos por su alejamiento, hay otros que no están menos vedados por su pequeñez. Como estos corpúsculos imperceptibles son las partes activas de la materia y los grandes instrumentos de la naturaleza, de la que dependen no sólo en todas sus cualidades secundarias, sino también la mayor parte de sus operaciones naturales, nuestra falta de ideas precisas y distintas sobre sus cualidades primarias nos mantiene en una ignorancia incurable respecto a lo que deseamos saber sobre ellos. No dudo que si

${ }^{28}$ Idem. 
pudiéramos descubrir la forma, el tamaño, la textura y el movimiento de las partes minúsculas constitutivas de dos cuerpos cualesquiera podríamos conocer sin necesidad de pruebas varias de sus operaciones, del mismo modo que conocemos las propiedades de un cuadrado o de un triángulo. Si conociéramos las propiedades mecánicas de las partículas del ruibarbo, de la cicuta, del opio y de un hombre de la misma manera que un relojero conoce un reloj, y por qué realiza sus operaciones, y conoce también las propiedades de una lima que, por su acción puede cambiar la forma de los mecanismos, seríamos capaces de afirmar que el ruibarbo purga a un hombre, que la cicuta lo mata y que el opio le produce somnolencia [...] Quizá entonces podríamos saber por qué se disuelve la plata en aqua fortis y el oro en aqua regia y no viceversa $[\ldots]^{29}$

\section{Conclusión}

Una de las cuestiones más interesantes de la reflexión lockeana sobre los universales es la de que en ella el filósofo inglés parece haber encontrado, gracias a la perspectiva mecanicista, una solución muy sugerente al debate sobre los universales, sobre el que sus antecesores medievales mantenían posiciones irreductibles o incompatibles, a saber: el nominalismo y el realismo. Como aparece en los textos citados, John Locke defiende ambas posturas y, por tanto, es nominalista y realista. Ahora bien, ¿cuál es la diferencia del filósofo con los representantes del realismo medieval? Yo pienso que ésta estriba en que el realismo de Locke se refiere a estructuras atómicas y no a esencias metafísicas. Las estructuras serán objeto de conocimiento de la filosofía natural, eso pensaba él en sus días y, en efecto, así lo es hoy, ya que actualmente la física y la química estudian las estructuras atómicas de los cuerpos físicos y no son objeto de una rama oscura de la filosofía, que es la metafísica, y contra la que luchaban todos los representantes de la "vía media" o escepticismo académico. En fin, creo que una de las grandes innovaciones del filósofo inglés, de la cual el Ensayo es un excelente ejemplo, es haber aplicado la hipótesis mecánica a problemas de la filosofía especulativa, como son la teoría del conocimiento y la metafísica, con lo cual sus respuestas a preguntas clásicas de la filosofía occidental resultan ser, por lo general, muy sorprendentes.

${ }^{29}$ Ibid., IV, iii, 25. 